\title{
Productividad científica profesional mediante indicadores de reconocimiento o visibilidad y de pertenencia repulsiva
}

\author{
Professional scientific productivity through indicators of recognition \\ or visibility and repulsive membership
}

Recibido: marzo 08 de 2019 | Revisado: mayo 20 de 2019 | Aceptado: julio 18 de 2019

\author{
George Argota Pérez ${ }^{\mathrm{I}}$ \\ Eusebio Aliaga Guillen ${ }^{2 A}$ \\ Josefa B. Pari Olarte ${ }^{2 \mathrm{~B}}$ \\ Cecilia G. Solano García ${ }^{2 \mathrm{C}}$ \\ Luz Chacaltana Ramos ${ }^{2 \mathrm{~B}}$ \\ Lida F. Velásquez SALCEdo ${ }^{2 C}$
}

\begin{abstract}
Resumen
El propósito del estudio fue valorar la productividad científica profesional mediante indicadores de reconocimiento o visibilidad y de pertenencia repulsiva. Se analizó la productividad científica mediante nueve indicadores agrupados en dos categorías: I) de reconocimiento o visibilidad y II) de pertenencia repulsiva. En la primera categoría se agrupó: publicación de artículo científico, libro o capítulo de libro, registro de patente e índice-H. En la segunda categoría se agrupó: número de proyecto, ponencia en eventos científicos, asesoría de tesis, curso de posgrado impartido y contribución directa al desarrollo empresarial. A cada indicador se le asignó un punto y al compararse los resultados entre dos investigadores (A y B) hubo diferencias estadísticamente significativas $(\mathrm{p}<0,05)$ donde el puntaje total resultó menor para el investigador $\mathrm{B}$, a pesar de presentar índice-H y no, el cumplimiento de otros indicadores. Se concluyó que, al mostrarse contribuciones sociales y no son aceptadas, existe pertenencia cognoscitiva repulsiva la cual desfavorece la productividad científica pudiendo ser la misma subjetiva solo si se considera el índice- $\mathrm{H}$.
\end{abstract}

Palabras clave: productividad cognoscitiva, impacto científico, indicador, resultados relevantes, publicación científica

\begin{abstract}
The purpose of the study was to assess professional scientific productivity through indicators of recognition or visibility and repulsive belonging. Scientific productivity was analysed using nine indicators grouped into two categories: I) of recognition or visibility and II) of repulsive belonging. In the first category it was grouped: publication of scientific article, book or book chapter, patent registration and H-index. In the second category was grouped: project number, presentation in scientific events, thesis advice, postgraduate course taught and direct contribution to business development. Each indicator was assigned a point and when comparing the results between two researchers (A and B) there were statistically significant differences $(p<0.05)$ where the total score was lower for researcher $B$, despite presenting $\mathrm{H}$-index and not, the compliance with other indicators. It was concluded that, by showing social contributions and not being accepted, there is repulsive cognitive belonging which disadvantages scientific productivity and can be the same subjective only if the H-index is considered.
\end{abstract}

Key words: cognitive productivity, scientific impact, indicator, relevant results, scientific publication

(C) Los autores. Este artículo es publicado por la Revista Campus de la Facultad de Ingeniería y Arquitectura de la Universidad de San Martín de Porres. Este artículo se distribuye en los términos de la Licencia Creative Commons Atribución No-comercial - Compartir-Igual 4.0 Internacional (https://creativecommons.org/licenses/ CC-BY), que permite el uso no comercial, distribución y reproducción en cualquier medio siempre que la obra original sea debidamente citada. Para uso comercial contactar a: revistacampus@usmp.pe. 


\section{Introducción}

La toma de decisiones es un proceso complejo, pues no solo depende de la competencia profesional con base a evidencias y prácticas (Luo, 2018) sino, en la calificación sobre la fuente de información donde se genera y analiza estadísticamente el dato, la interpretación requerida (Schwartz, 2016), además, del tipo de documento analizado en cada circunstancias pudiendo ser los trabajos de investigación (Ni \& An, 2018), proyectos de investigación (Shao et al., 2018), patentes otorgadas de investigación (Han $\&$ Magee, 2018) entre otros.

Otro de los factores que influye en la toma de decisiones radica en el grado de compromiso por parte de los colaboradores. Hu, Chen\&Liu (2014), señalan el grado de vinculación que puede existir durante la fase inicial, intermedia y final en cualquier proceso que se reconozca como productivo. La Figura 1 muestra lo planteado. Asimismo, Zhou\&Tian (2014) destacan que el tiempo de colaboración dedicado determina la productividad en el proceso de investigación y esto puede ser diferente cuando la participación de actores determina su condición de autoría (Hilário \&Grácio, 2017) o nivel de especialización (Dubois, Rochet \&Schlenker, 2014).

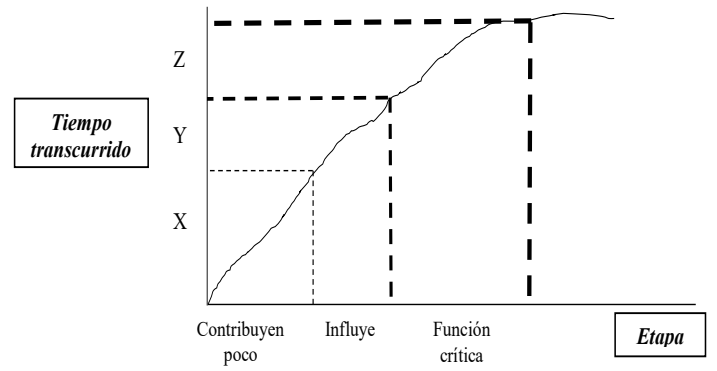

Figura 1. Compromiso profesional sobre el proceso de investigación científica.
Diferentes métodos se han desarrollado para evaluar el impacto de las investigaciones científicas y donde Taşkın\& Al (2018) destacan que pudiera ser relevante, solo el análisis de citas basadas en el contenido del documento, el análisis de las intenciones de citación y el análisis de la importancia de la cita, pues algunos investigadores como Macroberts \& Macroberts, (2018) argumentan que, existen ciertos sesgos cuando las citas de referencias, no reflejan el impacto de la información que trata el documento.

Tyrrell et al., (2017) y Ayaz, Masood\& Islam (2018) indican que el índice $\mathrm{H}$ (Hirsch, 2005) puede excluir la no relevancia de las referencias ya que demuestran el impacto de las investigaciones desde el número de artículos publicados y las veces que estos se citan, aunque del mismo modo, algunos autores critican no estar de acuerdo con este índice porque quizás no considera el campo de estudio y el tiempo de recorrido profesional (Aoun et al., 2013; Bornmann, 2014).

Por lo general, la producción científica solo es valorada como aquel resultado basado en la investigación científica y técnica sin considerar que, otros indicadores pueden favorecer el desarrollo social, a partir de la competencia profesional y su contribución. El propósito del estudio fue valorar la productividad científica profesional mediante indicadores de reconocimiento o visibilidad y de pertenencia repulsiva.

\section{Materiales y métodos}

Para analizar la productividad científica se consideró nueve indicadores agrupados en dos categorías: I) de reconocimiento o visibilidad y II) de pertenencia repulsiva. 
En la primera categoría se agrupó: publicación del artículo científico (cuartil de la revista indexada), libro o capítulo de libro, registro de patente (carácter nacional e internacional), índice-H. En la segunda categoría se agrupó: número de proyecto (concursable y no concursable), ponencia en eventos científicos (nacionales e internacionales), asesoría de tesis (diploma o grado, diplomado, especialidad, maestría, doctorado), curso de posgrado impartido (diplomado, especialidad, maestría, doctorado) y contribución directa al desarrollo empresarial (local, nacional, internacional). A cada indicador se les asignó un punto y luego se comparó el total alcanzado entre dos profesionales investigadores (A y B). El profesional A, afirmó cinco años de experiencia profesional mientras que, el profesional $\mathrm{B}$ certificó 10 años.

El análisis de los datos fue a través del programa estadístico Statgraphics Centurion 18 donde la normalidad se realizó mediante la prueba de Kolmogorov-S. La prueba t-Student se consideró en la comparación de medias siendo significativos los resultados cuando $\mathrm{p}<0,05$.

\section{Resultados}

La Tabla 1 muestra la puntuación de la productividad científica de los investigadores según los indicadores donde hubo diferencias estadísticamente significativas $(\mathrm{p}<0,05)$.

Tabla 1

Puntuación de la productividad cientifica / investigadores

\begin{tabular}{|c|c|c|c|c|}
\hline Categoría & Indicador & Clasificación & Investigador A & Investigador B \\
\hline \multirow{8}{*}{ I } & \multirow{4}{*}{ PCA } & Q1 & - & 1 \\
\hline & & Q2 & - & 1 \\
\hline & & Q3 & 1 & - \\
\hline & & Q4 & 1 & - \\
\hline & L-CP & Registrado & 1 & - \\
\hline & \multirow{2}{*}{$\mathrm{RP}$} & Nacional & - & - \\
\hline & & Internacional & - & - \\
\hline & $\mathrm{I}-\mathrm{H}$ & - & - & 1 \\
\hline \multirow{11}{*}{ II } & \multirow{2}{*}{ NP } & Concursable & - & 1 \\
\hline & & No concursable & 1 & 1 \\
\hline & \multirow{2}{*}{ PE } & Nacional & 1 & 1 \\
\hline & & Internacional & 1 & 1 \\
\hline & \multirow{4}{*}{ CPI } & Diplomado & - & - \\
\hline & & Especialidad & 1 & - \\
\hline & & Maestría & 1 & - \\
\hline & & Doctorado & 1 & 1 \\
\hline & \multirow{3}{*}{ CDDE } & Local & 1 & - \\
\hline & & Nacional & 1 & 1 \\
\hline & & Internacional & 1 & - \\
\hline \multicolumn{3}{|c|}{ Total } & 12 & 9 \\
\hline
\end{tabular}

Leyenda: publicación de artículo científico (PAC), libro (L), registro de patente (RP), índice-H, número de proyecto (NP), ponencia en evento (PE), asesoría de tesis (AT), curso posgrado impartido (CPI), contribución directa al desarrollo empresarial (CDDE) 


\section{Comparación de medias}

- Intervalos de confianza del 95,0 $\%$ para la media de investigador A: $12,0+/-0,2[11,8 ; 12,3]$

- Intervalos de confianza del 95,0\% para la media de investigador B: $9,0+/-0,3[8,8 ; 9,3]$

- Suponiendo varianzas iguales: 3,0 $+/-0,2[2,8 ; 3,2]$

\section{Prueba t para comparar medias}

- Hipótesis nula: media $1=$ media 2

- Hipótesis Alt.: media1 <> media2

- Suponiendo varianzas iguales: $\mathrm{t}=$ 36,74 valor-P $=0,000003$

Puesto que el intervalo no contiene el valor 0 , existe diferencia estadísticamente significativa entre las medias (puntuaciones de los investigadores) con un nivel de confianza del 95,0\%.

\section{Discusión}

Como pudo observarse, el investigador B presentó como indicador de reconocimiento o visibilidad un índice- $\mathrm{H}$ dado por sus publicaciones en revistas indexadas para Q1 como Q2, sin embargo, no presentó autorías en la publicación de libros o capítulos de libros. Es posible que, el investigador $\mathrm{A}$ al no presentar índice-H no sea reconocido, pues Wildgaard, Schneider \& Larsen (2014), señalan que, este indicador al medir el desempeño individual, entonces resultará evidente asumir limitada contribución a la ciencia cuando su valor sea bajo.

Van den Besselaar (2012), Jang et al., (2016) y Bagues et al., (2017) refieren que, la evaluación científica ha demostrado ser sesgada, pues está documentado que algunos revisores, condicionan cierto favorecimiento. Al analizar el número de publicaciones de artículos científicos entre los investigadores, la resultante fue la misma pero una pregunta quizás podría surgir: ¿de qué factores depende la selección de la revista para comunicar los resultados cientificos?

Si asumimos que al revisar el manuscrito fue aceptada la redacción, el constructivismo de la información, la aplicación de métodos y técnicas, presentación y contraste de los resultados, además, de excelente bibliografía consultada entonces que condujo a orientar la publicación en una revista cuyo factor de impacto fue menor y aunque así resultó, no debe asumirse como poco relevante el trabajo por su poca pretensión durante la selección de la revista.

Asimismo, en la categoría II, a pesar que el investigador A mostró como indicador de pertenencia repulsiva diferenciable comparativamente el proyecto concursable, otros indicadores para ambas categorías no cumplió por cuanto alcanzó menos puntaje lo cual indica que, aceptar como principal indicador la publicación de artículo científico, pudiera quizás ser un criterio subjetivo para la productividad científica en los profesionales.

De igual modo, cómo valorar al investigador A que contribuye social a la formación de profesionales, pues de forma conjunta a la impartición de cursos para programas de doctorado (especialista B), igualmente lo realiza en maestría y especialización. El investigador A posee más contribuciones directas al desarrollo social donde algunos resultados incluso pueden expresarse a mediano y largo plazo según el pensamiento estratégico de la empresa, donde Wong \& Singh (2013) 
señalan el impacto positivo que puede generarse ante la colaboración académica, además, la innovación de productos (Belderbos et al., 2016; McKelvey \& Rake, 2016) y el fortalecimiento que subyace al establecerse el consorcio empresa y otros actores que posibilitan potenciar la investigación y el desarrollo (García, Madrid \& Martin, 2017).

\section{Conclusión}

Un aspecto en la comparación entre los investigadores resultó significativo fue el puntaje alcanzado por el investigador A, quien lo consiguió durante la mitad de años de experiencia acumulada con relación al investigador B. Este resultado de ninguna manera puede ser considerado como subjetivo por la comunidad científica, pues un calificativo caracteriza al investigador A es el compromiso responsable con la ciencia de lo contrario, el cumplimiento de cualquier indicador "no aceptado" aunque muestren contribuciones sociales representa una pertenencia repulsiva.

\section{Referencias}

Aoun, S. G., Bendok, B. R., Rahme, R. J., Dacey, R. G. \&Batjer, H. H. (2013). Standardizing the evaluation of scientific and academic performance in neurosurgery-Critical review of the " $h$ " index and its variants. World Neurosurgery; 80(5), 8590 . https://doi.org/10.1016/j. wneu.2012.01.052

Ayaz, S., Masood, N. \& Islam, M.A. (2018). Predicting scientific impact based on h -index. Scientometrics; 114(3), 993-1010.https://doi. org/10.1007/s/11192-017-2618-1

Bagues, M., Sylos, L.M. \&Zinovyeva, N. (2017). Does the gender composition of scientific committees matter?.Am. Econ. Rev.; 107(4), 1207-1238. https://doi. org/10.1257/aer.20151211

Belderbos, R., Gilsing, V.A. \& Suzuki, S. (2016). Direct and mediated ties to universities: "scientific" absorptive capacity and innovation performance of pharmaceutical firms. Strateg. Organ; 14(1), 32-52. https://doi.org/10.1177\%2F14761 27015604734

Bornmann, L. (2014). h-Index research in scientometrics: A summary. Journal of Informetrics; 8(3), 749750. https://doi.org/10.1016/j. joi.2014.07.004

Dubois, P., Rochet, J.C. \&Schlenker, J.M. (2014). Productivity and mobility in academic research: Evidence from mathematicians. Scientometrics; 98(3), 1669-1701.https://doi. org/10.1007/s1119-013-1112-7

García, P.D., Madrid, G.A. \& Martin, D.P. (2017). Influence of university-firm governance on SMEs innovation and performance levels. Technol. Forecast. Soc. Chang, 123, 250261. https://doi.org/10.1016/j. techfore.2016.04.003

Han, F. \& Magee, C.L. (2018). Testing the science/technology relationship by analysis of patent 
citations of scientific papers after decomposition of both science and technology. Scientometrics; 116(2), 767-796. https://arxiv.org/ftp/ arxiv/papers/1705/1705.00258.pdf

Hilário, C.M. \&Grácio, M.C.C. (2017). Scientific collaboration in Brazilian researches: A comparative study in the information science, mathematics and dentistry fields. Scientometrics; 113(1), 1-22. https://doi.org/10.1007/s11192017-2498-4

Hirsch, J.E. (2005). An index to quantify an individual's scientific research output. Proceedings of the national Academy of Sciences of the United States of America; 102(46), 1656916572. https://doi.org/10.1073/ pnas. 0507655102

Hu, Z., Chen, C. \& Liu, Z. (2014). How are collaboration and productivity correlated at various career stages of scientists?.Scientometrics; 101(2), 1553-1564.https://doi. org/10.1007/s11192-014-1323-6

Jang, D., Doh, S., Kang, G.M. \& Han, D.S. (2016). Impact of alumni connections on peer review ratings and selection Success rate in national research. Sci. Technol. HumanValues; 42(1), 116-143. https://doi. org/10.1177/0162243916665466

Luo, L. (2018). Experiencing evidencebased library and information practice (EBLIP): Academic Librarians' perspective. College \& Research Libraries; 79(4), 554567. https://doi.org/10.5860/ crl.79.4.554
Macroberts, M.H. \&Macroberts, B.R. (2018). The mismeasure of science: Citation analysis. Journal of the Association for Information Science \& Technology; 69. http://dx.doi. org/10.1002/asi.23970

McKelvey, M. \& Rake, B. (2016). Product innovation success based on cancer research in the pharmaceutical industry: co-publication networks and the effects of partners. Ind. Innov; 23(5), 383-406. https:// doi.org/10.1080/13662716.2016. 1150157

Ni, P. \&An, X. (2018). Relationship between international collaboration papers and their citations from an economic perspective. Scientometrics; 116(2), 863-877. https://doi.org/10.1007/s11192018-2784-9

Schwartz, M. (2016). Top skills for tomorrow's librarians. https:// www.libraryjournal. com/?detailStory=top-skills-fortomorrows-librarians-careers 2016

Shao, Z.Y., Li, Y. M., Hui, F., Zheng, Y. \&Guo, Y.J. (2018). Interdisciplinarity research based on NSFC-sponsored projects: A case study of mathematics in Chinese universities. PLoS One; 13(7), 1-19. https://doi.org/10.1371/journal. pone. 0201577

Taşkın, Z. \& Al, U. (2018). A contentbased citation analysis study based on text categorization. Scientometrics; 114(4), 1-23. https://doi.org/10.1080/14683857 .2018 .1424888 
Tyrrell, P.N., Moody, A.R., Moody, J.O.C. \&Ghiam, N. (2017). Departmental h-index: Evidence for publishing less?.Canadian Association of Radiologists Journal; 68(1), $10-$ 15. https://doi.org/10.1016/j. carj.2016.05.005

Van den Besselaar, P. (2012). Selection committee membership: service or self-service. J. Informetr; 6(4), 580585. https://doi.org/10.1016/j. joi.2012.05.003

Wildgaard, L., Schneider, J. W. \& Larsen, B. (2014). A review of the characteristics of 108 authorlevel bibliometric indicators.
Scientometrics; 101(1), 125-158. https://doi.org/10-1007/s11192014-1423-3

Wong, P.K. \& Singh, A. (2013). Do co-publications with industry lead to higher levels of university technology commercialization activity?.Scientometrics; $\quad 97(2)$, 245-265. https://doi.org/10-1007/ s11192-013-1029-1

Zhou, P. \&Tian, H. (2014). Funded collaboration research in mathematics in China. Scientometrics; 99(3), 695-715. https://doi.org/10-1007/s11192013-1212-4 
